\title{
New Theoretical Analysis of the LRRM Calibration Technique for Vector Network Analyzers
}

\author{
Francesc Purroy and Lluís Pradell
}

\begin{abstract}
In this paper, a new theoretical analysis of the fourstandards line-reflect-reflect-match (LRRM) vector network-analyzer (VNA) calibration technique is presented. As a result, it is shown that the reference-impedance (to which the LRRM calibration is referred) cannot generally be defined whenever nonideal standards are used. Based on this consideration, a new algorithm to determine the on-wafer match standard is proposed that improves the LRRM calibration accuracy. Experimental verification of the new theory and algorithm using on-wafer calibrations up to $40 \mathrm{GHz}$ is given.
\end{abstract}

Index Terms-Calibration algorithms, calibration standards, calibration techniques, network-analyzer, on-wafer calibration, reference impedance, self-calibration.

\section{INTRODUCTION}

$\mathbf{I}$ $\mathrm{T}$ is well known that the systematic errors associated to a vector network-analyzer (VNA) can be modeled by error port adapters. The widely-used eight-term error model (Fig. 1) was first proposed in [1] and intended for two-way measurements. To compute its error terms, a calibration procedure is required. A number of calibration techniques have been proposed in the literature. A technique that requires measurements from only three standards was first proposed in [2] (thru-short-delay, or TSD, technique). A more general approach (thru-reflect-line, or TRL, technique), in which the short standard is replaced by a highly reflective, but unknown, standard (reflect), was proposed in [3], and fully developed in [4]. Self-calibration techniques, which take full advantage of the redundancy in the calibration process, were developed in [5] and generalized in [6], [7] (Txx, Lxx). A particular case is line-reflect-match (LRM). Improvements in the computation algorithm of these techniques can be found in [8]. A variation of LRM (LRM-known Reflect) is described in [9].

The LRM technique requires two match (matched load) standards, one at each VNA port. As originally proposed in [6], [7], both match standards should be equal and perfectly known. Since those requirements are not fulfilled in practice, the LRM calibration accuracy is reduced. The four-standards LRRM (line-reflect-reflect-match) calibration technique was proposed (but not mathematically developed) in [10], as an improvement with respect to LRM. The main advantage of

Manuscript received April 11, 1997; revised May 31, 2001. This work was supported by Research Projects TIC92-1020-C02-02, TIC93-0672-C04-04, and TIC97-1129-C04-04 and financed by the Spanish Science and Technology Commission (CICYT)

F. Purroy is with Ericsson Radio Access AB, Stockholm, Kista, Sweden.

L. Pradell is with the Universitat Politècnica de Catalunya (UPC), Departamento TSC, Campus Nord UPC-Modul D3, Barcelona, Catalunya, Spain.

Publisher Item Identifier S 0018-9456(01)08109-8.

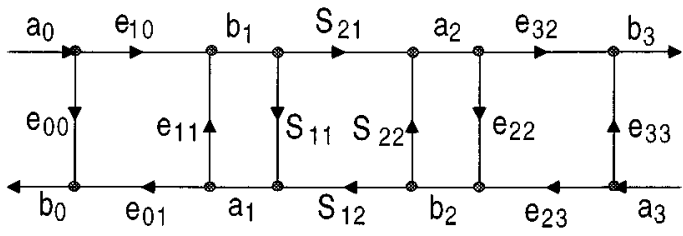

(a)

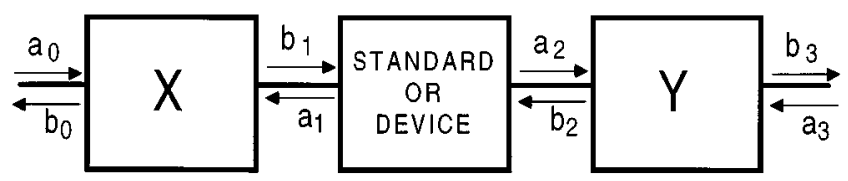

(b)

Fig. 1. Error model for a vector network analyzer: (a) eight-term error model and (b) error matrices.

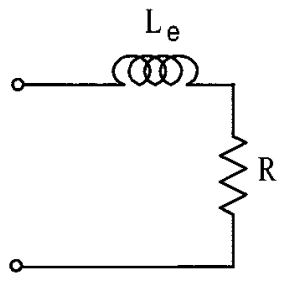

Fig. 2. Circuit model for on-wafer "match" standards.

LRRM versus LRM is that only one match standard (partially unknown) is required to accurately compute the two unknown reflect standards $\Gamma_{r 1}$ and $\Gamma_{r 2}$, avoiding problems due to asymmetries between both match standards. The on-wafer match is modeled by a perfectly known resistor in series with an unknown inductance $L_{\mathrm{e}}$ (see Fig. 2). The key point in the LRRM technique is an accurate determination of inductance $L_{\mathrm{e}}$ from the computed reflection coefficient, $\Gamma_{r 1}^{e}$, of a measured reflect standard. In [10] a simple expression to compute $L_{\mathrm{e}}$ from $\Gamma_{r 1}^{e}$ is proposed [expr. (3) of [10]]. In this expression, it is implicitly assumed that a reference-impedance (to which the calibration is referred) does exist and equals the match impedance. However, this assumption is not justified, and its impact on the determination of $L_{\mathrm{e}}$ is not considered.

In this paper, a full theory for LRRM is developed. To the authors' knowledge, a rigorous analysis of LRRM has not been published yet. Based on this theory [14], it is shown that the 'reference-impedance' (to which the LRRM calibration is referred) cannot generally be defined whenever nonideal standards are used. This consideration leads to a general expression 
for $\Gamma_{r 1}^{e}$ that, in contrast with (3) of [10], does not assume a reference-impedance. The expression is used to accurately determine $L_{\mathrm{e}}$, demonstrating accuracy improvements in the calibration.

\section{THEORY OF LRRM SELF-CALIBRATION TECHNIQUE}

In this section, the three-standards self-calibration theory [6] is generalized to the four-standards case, in particular for the LRRM technique. It is assumed throughout this paper that the VNA systematic errors can be modeled by the bidirectional eight-term error model given in Fig. 1. The error terms are unknown and have to be determined through the calibration procedure. Four error terms $\left(e_{00}, e_{01}, e_{10}, e_{11}\right)$ model the systematic errors corresponding to port 1 of the VNA (error-box $X$ ), while $e_{22}, e_{23}, e_{32}$, and $e_{33}$ model the systematic errors corresponding to port 2 of the VNA (error-box $Y$ ). It is assumed that isolation terms (terms that account for direct transmission between port 1 and port 2) are very small in practice and can be neglected (i.e., $\left.e_{02}=e_{20}=e_{12}=e_{21}=e_{03}=e_{30}=e_{13}=e_{31}=0\right)$. Error-boxes are described by their transmission matrices ( $T$-matrices) $[X],[Y]$, respectively, defined as

$$
\begin{aligned}
& {[X]=\frac{1}{e_{10}} \cdot[\bar{X}]=\frac{1}{e_{10}} \cdot\left(\begin{array}{cc}
e_{10} \cdot e_{01}-e_{00} \cdot e_{11} & e_{00} \\
-e_{11} & 1
\end{array}\right)} \\
& {[Y]=\frac{1}{e_{32}} \cdot[\bar{Y}]=\frac{1}{e_{32}} \cdot\left(\begin{array}{cc}
e_{32} \cdot e_{23}-e_{22} \cdot e_{33} & e_{22} \\
-e_{33} & 1
\end{array}\right)}
\end{aligned}
$$

where $[\bar{X}],[\bar{Y}]$ are normalized versions of $[X],[Y]$, respectively.

\section{A. Equation-Systems for the Error Matrices $[X]$ and $[Y]$}

First, the calibration standards used in the LRRM technique are defined and their corresponding transmission-matrices ( $T$-matrices) are given.

1) $\boldsymbol{L I N E}$ - Transmission line with a known length, propagation constant and characteristic impedance

$$
\left[T_{1}\right]=\left(\begin{array}{cc}
e^{-\gamma \cdot l} & 0 \\
0 & e^{\gamma \cdot l}
\end{array}\right)
$$

2) $\boldsymbol{R E F L E C T 1 - D u a l ~ o n e - p o r t ~ d e v i c e ~ m a d e ~ u p ~ b y ~ t w o ~}$ identical, isolated loads with a reflection coefficient $\Gamma_{r 1}$. Its magnitude is high but unknown and its phase must be known within $\pm \pi / 2$ radians

$$
\left[T_{2}\right]=\frac{1}{S_{21}^{r 1}} \cdot\left(\begin{array}{cc}
-\Gamma_{r 1}^{2} & \Gamma_{r 1} \\
-\Gamma_{r 1} & 1
\end{array}\right)
$$

where $S_{21}^{r 1}(=0)$ is the transmission $S$-parameter of the standard reflect1.

3) $\boldsymbol{R E F L E C T 2 ~ - ~ D u a l ~ o n e - p o r t ~ d e v i c e ~ m a d e ~ u p ~ b y ~ t w o ~}$ identical, isolated loads with a reflection coefficient $\Gamma_{r 2}$. Its magnitude is high but unknown and its phase must be known within $\pm \pi / 2$ radians

$$
\left[T_{3}\right]=\frac{1}{S_{21}^{r 2}} \cdot\left(\begin{array}{cc}
-\Gamma_{r 2}^{2} & \Gamma_{r 2} \\
-\Gamma_{r 2} & 1
\end{array}\right)
$$

where $S_{21}^{r 2}(=0)$ is the transmission $S$-parameter of the standard reflect2.
To obtain independent measurements, a nonideal opencircuit is used as reflect 1 and a nonideal short-circuit as reflect 2 .

4) $\boldsymbol{M A T C H}$-One-port device with an unknown reflection coefficient $\Gamma_{m}$. Its magnitude should be very small. As reflect1, 2 and match are nontransmission devices, it is possible to define four virtual standards combining the match (port 1 or 2 ) with reflect1 (port 2 or 1 ) or reflect 2 (port 2 or 1). If, for example, the match standard is connected to port 1 , the following two standards are defined.

MATCH-REFLECT1:

$$
\left[T_{4}\right]=\frac{1}{S_{21}^{m r 1}} \cdot\left(\begin{array}{cc}
-\Gamma_{r 1} \cdot \Gamma_{m} & \Gamma_{m} \\
-\Gamma_{r 1} & 1
\end{array}\right)
$$

where $S_{21}^{m r 1}(=0)$ is the transmission $S$-parameter of the standard match-reflect1.

MATCH-REFLECT2:

$$
\left[T_{5}\right]=\frac{1}{S_{21}^{m r 2}} \cdot\left(\begin{array}{cc}
-\Gamma_{r 2} \cdot \Gamma_{m} & \Gamma_{m} \\
-\Gamma_{r 2} & 1
\end{array}\right)
$$

where $S_{21}^{m r 2}(=0)$ is the transmission $S$-parameter of the standard match-reflect 2 .

For each standard $\left[T_{i}\right]$, a measurement transmission-matrix $M_{i}(i=1,2,3,4,5)$ is defined as (see (A3) in Appendix A)

$$
\left[M_{i}\right]=[\bar{X}] \cdot\left[T_{i}\right] \cdot[\bar{Y}] \frac{1}{e_{10} e_{32}} .
$$

Combining measurement matrix $\left[M_{1}\right]$ from standard 1 (line), with measurement matrix $\left[M_{i}\right]$ from another standard $i(i=$ $2,3,4,5)$, the following linear-equation systems for matrices $[\bar{X}]$ and $[\bar{Y}]$ are obtained from (7)

$$
\begin{aligned}
{\left[M_{i}\right] \cdot\left[M_{1}\right]^{-1} \cdot[\bar{X}] } & =[\bar{X}] \cdot\left[T_{i}\right] \cdot\left[T_{1}\right]^{-1} \\
{\left[M_{i}\right] \cdot\left[M_{1}\right]^{-1} \cdot[\bar{Y}] } & =[\bar{Y}] \cdot\left[T_{i}\right] \cdot\left[T_{1}\right]^{-1} \\
(i & =2,3,4,5)
\end{aligned}
$$

or, in a more compact form

$$
\begin{aligned}
{\left[M_{i 1}\right] \cdot[\bar{X}] } & =[\bar{X}] \cdot\left[T_{i 1}\right] \\
{\left[M_{i 1}\right] \cdot[\bar{Y}] } & =[\bar{Y}] \cdot\left[T_{i 1}\right] \\
(i & =2,3,4,5)
\end{aligned}
$$

where $\left[M_{i 1}\right]$ and $\left[T_{i 1}\right]$, are defined as

$$
\begin{aligned}
{\left[M_{i 1}\right] } & =\left[M_{i}\right] \cdot\left[M_{1}\right]^{-1} \quad(i=2,3,4,5) \\
{\left[T_{i 1}\right] } & =\left[T_{i}\right] \cdot\left[T_{1}\right]^{-1} \quad(i=2,3,4,5) .
\end{aligned}
$$

In (8)-(11), matrices $\left[M_{i}\right](i=1,2,3,4,5)$ are obtained from the measured $[S]$-parameters of standards [see (A5) in Appendix A], matrices $\left[T_{i}\right](i=1,2,3,4,5)$, partially unknown, are given by (2)-(6), and $[\bar{X}]$ and $[\bar{Y}]$ are the unknown error-matrices. Equation (9) express a similarity transformation between matrices $\left[M_{i 1}\right]$ and $\left[T_{i 1}\right]$, with the two following mathematical properties.

1) Trace conservation:

$$
T_{i 1}^{11}+T_{i 1}^{22}=M_{i 1}^{11}+M_{i 1}^{22}
$$


2) Determinant conservation:

$$
\operatorname{det}\left(\left[T_{i 1}\right]\right)=\operatorname{det}\left(\left[M_{i 1}\right]\right)
$$

Substituting (2)-(6) into (11) and the result into (12), we obtain

$$
\begin{aligned}
& \frac{1}{S_{21}^{r 1}}=\frac{M_{21}^{11}+M_{21}^{22}}{\left(-e^{\gamma \cdot l_{1}} \cdot \Gamma_{r 1}^{2}+e^{-\gamma \cdot l_{1}}\right)} \\
& \frac{1}{S_{21}^{r 2}}=\frac{M_{31}^{11}+M_{31}^{22}}{\left(-e^{\gamma \cdot l_{1}} \cdot \Gamma_{r 2}^{2}+e^{-\gamma \cdot l_{1}}\right)} \\
& \frac{1}{S_{21}^{m r 1}}=\frac{M_{41}^{11}+M_{41}^{22}}{\left(-e^{\gamma \cdot l_{1}} \cdot \Gamma_{r 1} \cdot \Gamma_{m}+e^{-\gamma \cdot l_{1}}\right)} \\
& \frac{1}{S_{21}^{m r 2}}=\frac{M_{\tilde{5} 1}^{11}+M_{51}^{22}}{\left(-e^{\gamma \cdot l_{1}} \cdot \Gamma_{r 2} \cdot \Gamma_{m}+e^{-\gamma \cdot l_{1}}\right)} \text {. }
\end{aligned}
$$

Equations (14) allow normalizing $\left[T_{i 1}\right]$ and $\left[M_{i 1}\right]$ to the same normalization factor to avoid the singularities of nontransmission standards $\left(S_{21}^{r 1}=S_{21}^{r 2}=S_{21}^{m r 1}=S_{21}^{m r 2}=0\right)$ in (3)-(7). In fact, using the normalized matrices $\left[\overline{M_{i}}\right]$ and $\left[\overline{T_{i}}\right](i=2,3,4,5)$ defined in Appendix A (A8), equation-systems (9) are written as

$$
\begin{aligned}
& {\left[\overline{M_{i 1}}\right] \cdot[\bar{X}]=[\bar{X}] \cdot\left[\overline{T_{i 1}}\right]} \\
& {\left[\overline{M_{i 1}}\right] \cdot[\bar{Y}]=[\bar{Y}] \cdot\left[\overline{T_{i 1}}\right]}
\end{aligned}
$$

where

$$
\begin{aligned}
{\left[\overline{M_{i 1}}\right] } & =\left[\overline{M_{i}}\right] \cdot\left[M_{1}\right]^{-1} \\
{\left[\overline{T_{i 1}}\right] } & =\left[\overline{T_{i}}\right] \cdot\left[T_{1}\right]^{-1} \\
{\left[\overline{T_{2}}\right] } & =\frac{\overline{M_{21}^{11}}+\overline{M_{21}^{22}}}{\left(-e^{\gamma \cdot l} \cdot \Gamma_{r 1}^{2}+e^{-\gamma \cdot l}\right)}\left(\begin{array}{cc}
-\Gamma_{r 1}^{2} & \Gamma_{r 1} \\
-\Gamma_{r 1} & 1
\end{array}\right) \\
{\left[\overline{T_{3}}\right] } & =\frac{\overline{M_{31}^{11}}+\overline{M_{31}^{22}}}{\left(-e^{\gamma \cdot l} \cdot \Gamma_{r 2}^{2}+e^{-\gamma \cdot l}\right)}\left(\begin{array}{cc}
-\Gamma_{r 2}^{2} & \Gamma_{r 2} \\
-\Gamma_{r 2} & 1
\end{array}\right) \\
{\left[\overline{T_{4}}\right] } & =\frac{\frac{M_{41}^{11}}{M_{41}^{22}}}{\left(-e^{\gamma \cdot l} \cdot \Gamma_{r 1} \cdot \Gamma_{m}+e^{-\gamma \cdot l}\right)}\left(\begin{array}{cc}
-\Gamma_{r 1} \Gamma_{m} & \Gamma_{m} \\
-\Gamma_{r 1} & 1
\end{array}\right) \\
{\left[\overline{T_{5}}\right] } & =\frac{\overline{M_{51}^{11}}+\overline{M_{51}^{22}}}{\left(-e^{\gamma \cdot l} \cdot \Gamma_{r 2} \cdot \Gamma_{m}+e^{-\gamma \cdot l}\right)}\left(\begin{array}{cc}
-\Gamma_{r 2} \Gamma_{m} & \Gamma_{m} \\
-\Gamma_{r 2} & 1
\end{array}\right)
\end{aligned}
$$

Note that, since $\left[\overline{M_{i 1}}\right]$ in (16) do not require the measurement of $S_{21}^{M_{i}}$, they are not singular; therefore, $\left[\overline{T_{i 1}}\right](17)-(21)$ are not singular. Equation system (15) can be rearranged to form a system of 4 linear-equations for the 3 elements of $[\bar{X}]$

$$
\begin{aligned}
& \left(\begin{array}{cccc}
\overline{T_{i 1}^{11}}-\overline{M_{i 1}^{11}} & \overline{T_{i 1}^{21}} & -\overline{M_{i 1}^{12}} & 0 \\
\frac{T_{i 1}^{12}}{M_{i 1}^{21}} & T_{i 1}^{22}-\overline{M_{i 1}^{11}} & 0 & -\overline{M_{i 1}^{12}} \\
0 & -\overline{M_{i 1}^{21}} & T_{i 1}^{11}-\overline{M_{i 1}^{22}} & \overline{T_{i 1}^{21}} \\
\overline{T_{i 1}^{22}}-\overline{M_{i 1}^{22}}
\end{array}\right) \\
& \times\left(\begin{array}{c}
\overline{X_{11}} \\
\frac{X_{12}}{X_{21}} \\
1
\end{array}\right)=\left(\begin{array}{l}
0 \\
0 \\
0 \\
0
\end{array}\right)
\end{aligned}
$$

A similar system of 4 linear-equations for the 3 elements of $[\bar{Y}]$ could also be written substituting $\overline{X_{11}}, \overline{X_{12}}, \overline{X_{21}}$ for $\overline{Y_{11}}$, $\overline{Y_{12}}, \overline{Y_{21}}$ in (22). Since 4 standards $(i=2,3,4,5)$ can be combined with standard 1 (thru), equation-system (22) provides 16 linear equations for $[\bar{X}]$ and [using $\overline{Y_{11}}, \overline{Y_{12}}, \overline{Y_{21}}$ in (22)], 16 linear equations for $[\bar{Y}]$. It can be shown (numerically) that the system composed by 16 equations has a maximum rank of 3 . Therefore, only 3 terms of matrix $[\bar{X}]$ can be determined, from which error-terms $e_{00}, e_{11}, e_{10} \cdot e_{01}$ are computed. The same holds for $[\bar{Y}]$ and $e_{22}, e_{33}, e_{23} \cdot e_{32}$. The other two error-terms required $\left(e_{10} e_{32}, e_{01} e_{23}\right)$ are computed from the measured matrix $\left[M_{1}\right]$ (standard 'Thru') and the results obtained for $e_{00}, e_{11}$, $e_{10} \cdot e_{01}$ and $e_{22}, e_{33,} e_{23} \cdot e_{32}$.

There are 560 possible systems of 3 equations in (22), from which 16 have rank 2 . The rest (544) have rank 3 . The criterion proposed to select a three-equation system for the determination of error-terms, is the system condition-number (CN). Using experimental calibration data, the $\mathrm{CN}$ for every system of rank 3 in (22) has been computed. The experimental set-up is composed by a Hewlett-Packard 8510B VNA and a Cascade-Microtech SUMMIT 9000 wafer-probe station, with its calibration substrate type LRM-ISS. Measurement frequency-range was 1 to $40 \mathrm{GHz}$. Two possibilities for connecting the match standard have been considered, match connected to calibration Port 2, and match connected to calibration Port 1. The following two best conditioned systems have been found.

Match standard connected to Port 1:

$$
\begin{aligned}
& \left(\begin{array}{ccc}
-\overline{M_{21}^{21}} & 0 & \overline{T_{21}^{11}}-\overline{M_{21}^{22}} \\
-\overline{M_{31}^{21}} & 0 & \overline{T_{31}^{11}}-\overline{M_{31}^{22}} \\
0 & -\overline{M_{41}^{21}} & \overline{T_{41}^{12}}
\end{array}\right) \cdot\left(\begin{array}{l}
\overline{X_{11}} \\
\frac{X_{12}}{X_{21}}
\end{array}\right) \\
& =\left(\begin{array}{c}
-\overline{T_{21}^{21}} \\
-\overline{T_{31}^{21}} \\
\overline{M_{41}^{22}}-\overline{T_{41}^{22}}
\end{array}\right) \\
& \left(\begin{array}{ccc}
\overline{T_{21}^{12}} & \overline{T_{21}^{22}}-\overline{M_{21}^{11}} & 0 \\
-\overline{M_{21}^{21}} & 0 & \overline{T_{21}^{11}}-\overline{M_{21}^{22}} \\
\overline{T_{51}^{12}} & \overline{T_{51}^{22}}-\overline{M_{51}^{11}} & 0
\end{array}\right) \cdot\left(\begin{array}{l}
\overline{Y_{11}} \\
\overline{Y_{12}} \\
\overline{Y_{21}}
\end{array}\right) \\
& =\left(\begin{array}{c}
\overline{M_{21}^{12}} \\
\frac{-\overline{T_{21}^{21}}}{M_{51}^{12}}
\end{array}\right) \text {. }
\end{aligned}
$$

Match standard connected to Port 2:

$$
\begin{aligned}
& \left(\begin{array}{ccc}
\overline{T_{41}^{12}} & \overline{T_{41}^{22}}-\overline{M_{41}^{11}} & 0 \\
0 & -\overline{M_{\tilde{2}}^{21}} & \overline{T_{51}^{12}} \\
\overline{T_{51}^{12}} & \overline{T_{51}^{22}}-\overline{M_{51}^{11}} & 0
\end{array}\right) \cdot\left(\begin{array}{l}
\overline{X_{11}} \\
\overline{X_{12}} \\
\overline{X_{21}}
\end{array}\right)
\end{aligned}
$$

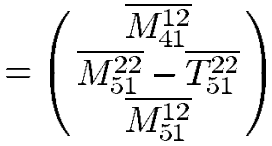

$$
\begin{aligned}
& \left(\begin{array}{ccc}
-\overline{M_{21}^{21}} & 0 & \overline{T_{21}^{11}}-\overline{M_{21}^{22}} \\
-\overline{M_{31}^{21}} & 0 & \overline{T_{31}^{11}}-\overline{M_{31}^{22}} \\
0 & -\overline{M_{51}^{21}} & \frac{\overline{T_{51}}}{\overline{Y_{11}}}
\end{array}\right) \cdot\left(\begin{array}{l}
\overline{Y_{12}} \\
\overline{Y_{21}}
\end{array}\right) \\
& =\left(\begin{array}{c}
-\overline{T_{21}^{21}} \\
-\overline{T_{31}^{21}} \\
\overline{M_{\check{2} 1}^{22}}-\overline{T_{\check{2} 1}^{22}}
\end{array}\right) .
\end{aligned}
$$




\section{B. Expressions for $\Gamma_{r 1}$ (Reflect1) and $\Gamma_{r 2}$ (Reflect 2$)$}

In this subsection, we focus on the determination of the unknown parameters $\Gamma_{r 1}$ (reflect1) and $\Gamma_{r 2}$ (reflect2), in (3)-(6), which are needed prior to solving systems (23) or (24). Using matrices $\left[T_{2}\right]$ and $\left[T_{3}\right]$ of standards reflect1 and reflect $2[(3),(4)$, respectively], two new combined normalized matrices, $\left[\overline{T_{32}}\right]=$ $\left[\overline{T_{3}}\right] \cdot\left[\overline{T_{2}}\right]^{-1}$ and $\left[\overline{M_{32}}\right]=\left[\overline{M_{3}}\right] \cdot\left[\overline{M_{2}}\right]^{-1}$, are defined. They can also be expressed in terms of $\left[\overline{T_{31}}\right],\left[\overline{T_{21}}\right]$ and $\left[\overline{M_{31}}\right],\left[\overline{M_{21}}\right]$ as

$$
\begin{aligned}
{\left[\overline{T_{32}}\right] } & =\left[\overline{T_{31}}\right] \cdot\left[\overline{T_{21}}\right]^{-1} \\
{\left[\overline{M_{32}}\right] } & =\left[\overline{M_{31}}\right] \cdot\left[M_{21}\right]^{-1} .
\end{aligned}
$$

Since these new matrices also fulfill trace and determinant conservation, (12) and (13) can be applied to (25) and (26) to obtain the following expression

$$
\frac{\overline{T_{21}^{11}}}{\overline{T_{21}^{22}}}+\frac{\overline{T_{31}^{11}}}{\overline{T_{31}^{22}}}-\frac{\overline{T_{31}^{21}} \cdot \overline{T_{21}^{12}}}{\overline{T_{31}^{22}} \cdot \overline{T_{21}^{22}}}-\frac{\overline{T_{31}^{12}} \cdot \overline{T_{21}^{21}}}{\overline{T_{31}^{22}} \cdot \overline{T_{21}^{22}}}=\frac{C_{31}^{21}}{\overline{T_{31}^{22}} \cdot \overline{T_{21}^{22}}}
$$

where

$$
\begin{array}{r}
C_{31}^{21}=\overline{M_{31}^{11}} \cdot \overline{M_{21}^{22}}-\overline{M_{31}^{12}} \cdot \overline{M_{21}^{21}} \\
-\overline{M_{31}^{21}} \cdot \overline{M_{21}^{12}}+\overline{M_{31}^{22}} \cdot \overline{M_{21}^{11}} .
\end{array}
$$

Substituting (18) and (19) into (27) and then using (12) again, the following equation for $\Gamma_{r 1}$ and $\Gamma_{r 2}$ is obtained

$$
\begin{gathered}
\Gamma_{r 2}^{2} \cdot\left(-1+K_{31}^{21} \cdot\left(1-\Gamma_{r 1}^{2} \cdot e^{2 \cdot \gamma \cdot l}\right)\right)+\Gamma_{r 2} \cdot\left(2 \cdot \Gamma_{r 1}\right) \\
+\Gamma_{r 1}^{2} \cdot\left(K_{31}^{21}-1\right)-K_{31}^{21} \cdot e^{-2 \cdot \gamma \cdot l}=0 .
\end{gathered}
$$

where

$$
K_{31}^{21}=\frac{C_{31}^{21}}{\left(\overline{M_{31}^{11}}+\overline{M_{31}^{22}}\right) \cdot\left(\overline{M_{21}^{11}}+\overline{M_{21}^{22}}\right) .}
$$

Repeating the above procedure for $\left[\overline{T_{52}}\right]=\left[\overline{T_{51}}\right] \cdot\left[\overline{T_{21}}\right]^{-1}$ and $\left[\overline{T_{43}}\right]=\left[\overline{T_{41}}\right] \cdot\left[\overline{T_{31}}\right]^{-1}$, the following expressions are obtained for $\Gamma_{r 2}$ and $\Gamma_{r 1}$, respectively

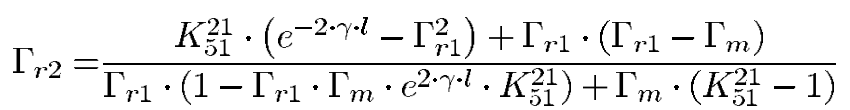

where

$$
\begin{aligned}
K_{51}^{21} & =\frac{\overline{M_{51}^{11}} \cdot \overline{M_{21}^{22}}-\overline{M_{51}^{12}} \cdot \overline{M_{21}^{21}}-\overline{M_{51}^{21}} \cdot \overline{M_{21}^{12}}+\overline{M_{51}^{22}} \cdot \overline{M_{21}^{11}}}{\left(\overline{M_{51}^{11}}+\overline{M_{51}^{22}}\right) \cdot\left(\overline{M_{21}^{11}}+\overline{M_{21}^{22}}\right)} \\
\Gamma_{r 1} & =\frac{K_{41}^{31} \cdot\left(e^{-2 \cdot \gamma \cdot l}-\Gamma_{r 2}^{2}\right)+\Gamma_{r 2} \cdot\left(\Gamma_{r 2}-\Gamma_{m}\right)}{\Gamma_{r 2} \cdot\left(1-\Gamma_{r 2} \cdot \Gamma_{m} \cdot e^{2 \cdot \gamma \cdot l} \cdot K_{41}^{31}\right)+\Gamma_{m} \cdot\left(K_{41}^{31}-1\right)}
\end{aligned}
$$

where

$$
=\frac{K_{41}^{31}}{\overline{M_{41}^{11}} \cdot \overline{M_{31}^{22}}-\overline{M_{41}^{12}} \cdot \overline{M_{31}^{21}}-\overline{M_{41}^{21}} \cdot \overline{M_{31}^{12}}+\overline{M_{41}^{22}} \cdot \overline{M_{31}^{11}}} .
$$

Substituting (29) [or (30)] into (28), a sixth degree equation for $\Gamma_{r 1}$ (for $\Gamma_{r 2}$ ) is derived. It can be shown that it has the following two double roots

$$
\Gamma_{r 1}= \pm e^{-\gamma \cdot l}\left(\Gamma_{r 2}= \pm e^{-\gamma \cdot l}\right) .
$$

The other two roots are computed solving the following seconddegree equation

$$
\left(\Gamma_{r 1}\right)^{2} \cdot a+\Gamma_{r 1} \cdot b+c=0
$$

where

$$
\begin{aligned}
\mathbf{a}= & K_{31}^{21} \cdot e^{-2 \cdot \gamma \cdot l} \cdot\left(2 \cdot K_{51}^{21}-1\right)-\left(K_{51}^{21}\right)^{2} \cdot \Gamma_{m}^{2} \cdot K_{31}^{21} \\
& \cdot\left(\frac{1}{K_{31}^{21}}+\frac{e^{-2 \cdot \gamma \cdot l}}{\Gamma_{m}^{2}}-1\right) \\
\mathbf{b}= & 2 \cdot \Gamma_{m}^{2} \cdot K_{31}^{21} \cdot e^{-2 \cdot \gamma \cdot l}\left(1-2 \cdot K_{51}^{21}+\frac{\left(K_{51}^{21}\right)^{2}}{K_{31}^{21}}\right) \\
\mathbf{c}= & \Gamma_{m}^{2} \cdot K_{31}^{21} \cdot e^{-2 \cdot \gamma \cdot l}\left(2 \cdot K_{51}^{21}-\left(K_{51}^{21}\right)^{2}-1\right) \\
& +\left(K_{31}^{21}-1\right) \cdot\left(K_{51}^{21}\right)^{2} \cdot e^{-4 \cdot \gamma \cdot l} .
\end{aligned}
$$

Equation (32) is used to compute $\Gamma_{r 1}$. It can also be used to compute $\Gamma_{r 2}$ by exchanging $\Gamma_{r 1}$ with $\Gamma_{r 2}$, and $K_{51}^{21}$ with $K_{41}^{31}$. To select the right root of (32) the phase of $\Gamma_{r 1}$ (or $\Gamma_{r 2}$ ) must be known within $\pm \pi / 2$ rad. Note that, prior to solving (32), $\Gamma_{m}$ should be known. An iterative method to compute $\Gamma_{m}$ is presented in the next subsection.

\section{Determination of $\Gamma_{m}$ (Match)}

According to [10], an on-wafer match standard can be modeled using the simple circuit of Fig. 2. Since the equivalent inductance $L_{\mathrm{e}}$ is unknown, an initial estimation is used for $\Gamma_{m}$, namely, $\Gamma_{m}=0$. This is equivalent to assuming $L_{\mathrm{e}}=0$ and $R=Z_{0}$, where $Z_{0}$ is the normalizing impedance (usually $50 \Omega$ ). Other values could be used for $R$, provided they are well known. When using on-wafer standards, some match elements are trimmed to a known accuracy (typically better than $\pm 2 \%$ ). Using $\Gamma_{m}=0$, (32) is solved for an initial estimation of $\Gamma_{r 1}$ (or $\Gamma_{r 2}$ ), $\Gamma_{r 1}^{e}, \Gamma_{r 2}^{e}$, respectively. The next step is to compute the actual value of $\Gamma_{m}$ by using $\Gamma_{r 1}^{e}$ (or $\Gamma_{r 2}^{e}$ ). In [10], an expression is given that relates the computed (measured) reflect admittance $\left(Y_{R}^{C}\right)$ to its actual value $\left(Y_{R}^{A}\right)$ whenever the match standard is improperly defined (expression (1) of [10])

$$
Y_{R}^{C}=\frac{Y_{M}^{D}}{Y_{M}^{A}} Y_{R}^{A}
$$

where $Y_{M}^{A}=1 / Z_{M}^{A}=1 /\left(R+j \omega L_{\mathrm{e}}\right)$ and $Y_{M}^{D}=1 / R$ are the actual and defined values for the match admittance, respectively. Equation (33) can be interpreted as a change in the calibration reference-impedance, from $R$ (defined value) to $Z_{M}^{A}=$ $R+j \omega L_{\mathrm{e}}$. In fact, substituting (33) into the expression for the computed reflection coefficient of standard reflect $\Gamma_{r}^{e}$

$$
\Gamma_{r}^{e}=\frac{Y_{o}-Y_{R}^{C}}{Y_{o}+Y_{R}^{C}}
$$


where $Y_{o}=Y_{M}^{D}=1 / R$ is the normalizing admittance, one obtains

$$
\Gamma_{r}^{e}=\frac{Y_{M}^{A}-Y_{R}^{A}}{Y_{M}^{A}+Y_{R}^{A}}
$$

Expression (35) can be interpreted as a change in the reference impedance to which an imperfect calibration is referred; imperfect means that some assumptions about the standards do not hold, in particular the actual and defined values for the match admittance are different. Therefore, $\Gamma_{r}^{e}$ (measured by the imperfect calibration) is referred to the equivalent reference impedance $Z_{M}^{A}$. The concept of reference impedance has been used in the literature to compare different calibrations [12], [13]. Obviously, a normalization impedance $\left(Z_{o}\right)$, to which the actual (or assumed) reflection coefficients are referred, does exist. As we show later in this subsection, a reference impedance may not be defined for some imperfect calibrations. An expression equivalent to (35) is [11]

$$
\Gamma_{r}^{e}=\frac{\Gamma_{M}-\Gamma_{R}}{1-\Gamma_{M} \Gamma_{R}}
$$

where $\Gamma_{M}=\left(Y_{o}-Y_{M}^{A}\right) /\left(Y_{o}+Y_{M}^{A}\right)$ and $\Gamma_{R}=$ $\left(Y_{o}-Y_{R}^{A}\right) /\left(Y_{o}+Y_{R}^{A}\right)$. In other words, (33) assumes that a reference impedance does exist and it is equal to the match impedance, $Z_{M}^{A}$. To compute $L_{\mathrm{e}}$, in [10] it is assumed that the real part of $Y_{R}^{A}$ in (33) is zero (this assumption is reasonable for on-wafer open standards). In this case, the following expression [(3) of [10]] is obtained from (33)

$$
L_{\mathrm{e}}=-\frac{G_{R}^{C} \cdot R}{B_{R}^{C} \cdot \omega}
$$

where $G_{R}^{C}$ and $B_{R}^{C}$ are real and imaginary parts of $Y_{R}^{C}$, respectively.

However, in an actual (nonideal) LRRM calibration the concept of calibration reference impedance may not be defined. Therefore, (33) and (37) cannot generally be applied. In fact, assume the following nonideal standards:

1) Nonideal line with (nonzero) physical length $(\ell \neq 0)$ and a characteristic impedance $Z_{\mathrm{L}}$ which is different from the normalizing impedance $Z_{o}$. A reflection coefficient $\rho_{\mathrm{L}}$ is defined for $Z_{\mathrm{L}}, \rho_{\mathrm{L}}=\left(Z_{\mathrm{L}}-Z_{o}\right) /\left(Z_{\mathrm{L}}+Z_{o}\right)$, different from zero. However, the LRRM algorithm assumes a perfectly matched line $\left(\rho_{\mathrm{L}}=0\right)$.

2) Symmetrical unknown reflect $\left(\Gamma_{r 1}\right)$.

3) Nonideal symmetrical match $\left(\Gamma_{m} \neq 0\right)$, but assumed ideal in the LRRM algorithm $\left(\Gamma_{m}=0\right)$.

As shown in Appendix B, the computed reflect reflection coefficient with this actual LRRM calibration is given by (B.4) [see equation (38) at the bottom of the page].
If $\ell=0$, then (38) reduces to

$$
\Gamma_{r 1}^{e}= \pm \frac{\left(\Gamma_{m}-\Gamma_{r 1}\right)}{\Gamma_{r 1} \cdot \Gamma_{m}-1} .
$$

Expression (38) shows that the actual reflection coefficient $\left(\Gamma_{r 1}\right)$ and the estimated $\left(\Gamma_{r 1}^{e}\right)$ values are not related by a change in the calibration reference impedance. Therefore, a reference impedance, to which the reflection coefficients computed by this imperfect calibration are referred, cannot be defined. In the particular case of zero-length line $(\ell=0)$, (39) holds, and the calibration reference impedance does exist and equals the actual match impedance $Z_{M}^{A}$.

The equivalent inductance in the match model can be computed using (38) assuming that the line standard is perfectly matched $\left(\rho_{\mathrm{L}}=0\right)$. In this case, $(38)$ reduces to

$$
\Gamma_{r 1}^{e}= \pm \frac{\Gamma_{r 1}-\Gamma_{m}}{e^{2 \cdot \gamma \cdot l} \cdot \Gamma_{m} \cdot \Gamma_{r 1}-1}
$$

where

$$
\Gamma_{m}=\frac{\left(w \cdot L_{\mathrm{e}}\right)^{2}+j \cdot 2 \cdot R \cdot\left(w \cdot L_{\mathrm{e}}\right)}{4 \cdot R^{2}+\left(w \cdot L_{\mathrm{e}}\right)^{2}} .
$$

Substituting (41) into (40) (using the negative sign) and enforcing $\left|\Gamma_{r 1}\right|=1$ (reflect assumed fully reactive [10]), a second-order equation for $\left(w \cdot L_{\mathrm{e}}\right)$ is obtained

$$
a \cdot\left(w \cdot L_{\mathbf{e}}\right)^{2}+b \cdot\left(w \cdot L_{\mathbf{e}}\right)+c=0 .
$$

where

$$
\begin{aligned}
a= & 2 \cdot \Re e\left(\Gamma_{r 1}^{e}\right)+\left|\Gamma_{r 1}^{e}\right|^{2}-2 \cdot \Re e\left(\Gamma_{r 1}^{e} \cdot e^{2 \cdot \gamma \cdot l}\right) \\
& -\left|\Gamma_{r 1}^{e} \cdot e^{2 \cdot \gamma \cdot l}\right|^{2} \\
b= & 4 \cdot R_{d c} \cdot\left(\Im m\left(\Gamma_{r 1}^{e}\right)+\Im m\left(\Gamma_{r 1}^{e} \cdot e^{2 \cdot \gamma \cdot l}\right)\right) \\
c= & 4 \cdot R_{d c}^{2} \cdot\left(\left|\Gamma_{r 1}^{e}\right|^{2}-1\right) .
\end{aligned}
$$

Equation (42) gives two solutions for the equivalent inductance $L_{\mathrm{e}}$. Equation (41) is used to select the solution giving the smallest $\left|\Gamma_{m}\right|$.

\section{Simulations}

To show the advantages of this new method, it is useful to simulate the LRRM calibration, and to compare results to those of the method in [10]. Two cases are considered, ideal line, and nonideal line.

\section{a) Ideal Line}

Line: Perfectly matched, 1 ps delay.

Reflect1: Symmetrical open-circuit, $C=-12 \mathrm{fF}$.

Reflect2: Symmetrical short-circuit, $L=6.244 \mathrm{pH}$.

Match: $R=50 \Omega$ and $L_{\mathrm{e}}=-7 \mathrm{pH}$.

Using the proposed calibration algorithm, $L_{\mathrm{e}}$ is computed and compared to the assumed value $\left(L_{\mathrm{e}}=-7 \mathrm{pH}\right)$, as shown in Fig. 3(a). Whereas the new method is giving

$$
\Gamma_{r 1}^{e}= \pm \frac{\left(\Gamma_{m}-\Gamma_{r 1}\right) \cdot\left(\left(\rho_{\mathrm{L}}\right)^{2}-1\right)}{e^{2 \cdot \gamma \cdot l} \cdot\left(\rho_{\mathrm{L}}-\Gamma_{r 1}\right) \cdot\left(\rho_{\mathrm{L}}-\Gamma_{m}\right)+\left(\Gamma_{r 1} \cdot \rho_{\mathrm{L}}-1\right) \cdot\left(1-\Gamma_{m} \cdot \rho_{\mathrm{L}}\right)}
$$



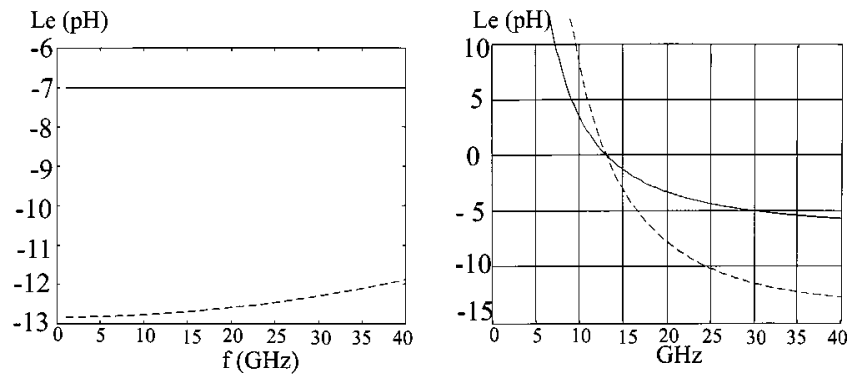

Fig. 3. Equivalent inductance $\left(L_{\mathrm{c}}\right)$ of a simulated "match" standard using the new method proposed in this paper (-) and the method proposed in [10] (-): (a) "line" perfectly matched and(b) "line" mismatched at low frequency (actual case).

the right value, the method in [10] is giving $L_{\mathrm{e}}$ ranging from $-12 \mathrm{pH}$ to $-13 \mathrm{pH}$ as a function of frequency. It can also be shown that the error increases when the assumed $\left|L_{\mathrm{e}}\right|$ increases.

\section{b) Nonideal Line}

Line: Coplanar-waveguide transmission line, with a delay of 1 ps. Its characteristic impedance, assumed $50 \Omega$ in the upper frequency range, was modeled using the expression for a lossy line with the following parameters

$$
\begin{aligned}
Z_{\mathrm{L}}^{a} & =\sqrt{\frac{R+j \cdot w \cdot L}{G+j \cdot w \cdot C}} \\
L & =386 \mathrm{nH} / \mathrm{m} \quad C=154 \mathrm{pF} / \mathrm{m} \\
G & =w \cdot C \cdot \operatorname{tg} \delta_{\mathrm{L}} \quad R \propto \sqrt{\frac{\pi \cdot f \cdot \mu_{o}}{\sigma_{\mathrm{C}}}}
\end{aligned}
$$

where $\operatorname{tg}\left(\delta_{\mathrm{L}}\right)$ is the dielectric (alumina) loss-tangent and $\sigma_{\mathrm{C}}$ is the conductor (gold) conductivity. The scale factor for $R$ was found by fitting (as a function of frequency) the loss-constant of the coplanar-waveguide line, that was obtained from a TRL calibration performed on-wafer.

Reflect1: Symmetrical open-circuit, $C=-7.19 \mathrm{fF}$.

Reflect2: Symmetrical short-circuit, $L=6.24 \mathrm{pH}$.

Match: $R=50 \Omega$ and $L_{\mathrm{e}}=-7 \mathrm{pH}$.

The result of this second simulation is shown in Fig. 3(b). At low frequencies, where the line standard is not well-matched, both methods get bad results, because the algorithm is assuming that the line is perfectly matched. At higher frequencies the results are similar to case (a).

\section{EXPERIMENTAL RESULTS}

The calibration algorithm proposed in this paper has been experimentally tested using the experimental set-up described in the preceding section. Details of the calibration standards and wafer-probes are

CALIBRATION SUBSTRATE: LRM-ISS (Cascade-Microtech).

Line: 1 ps delay line assumed perfectly matched.

Reflect1: Open circuit (probe tips in air) assumed symmetrical.
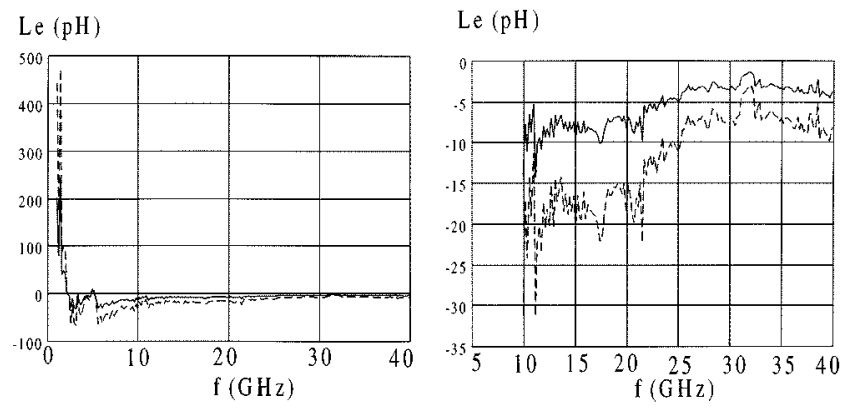

Fig. 4. Equivalent inductance $\left(L_{c}\right)$ of a measured "match" standard computed using the new method (-) and the method in [10] (-).

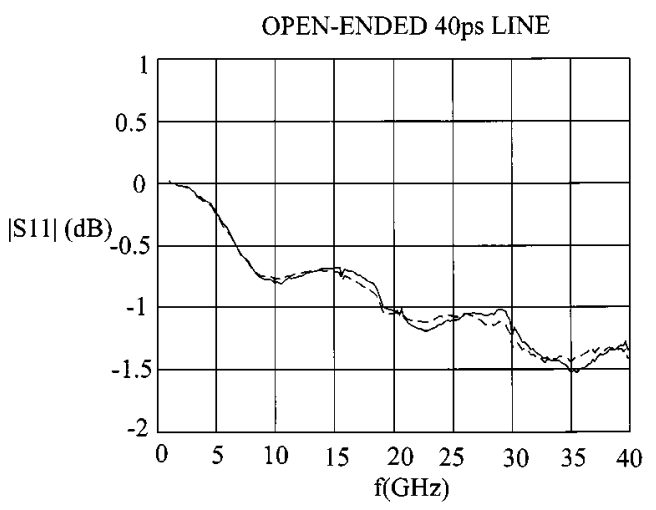

Fig. 5. Measured $\left|S_{11}\right|$ of a 40 ps-delay open-ended coplanar-waveguide line using LRRM (-) and LRM (-)

Reflect2: Short circuit assumed symmetrical.

Match: Coplanar load, $R=50 \Omega$.

WAFER-PROBES: WPH-305-150 (Cascade-Microtech).

Fig. 4 shows the equivalent inductance $L_{\mathrm{e}}$ computed using the method proposed in [10] as well as using the new method proposed in this paper. Above $10 \mathrm{GHz}, L_{\mathrm{e}}$ is more frequency-independent when using the new method. In the low-frequency margin, both methods compute incorrect values, because the line standard is not well matched. These results are in agreement with predictions of simulations described in Section III (see Fig. 3).

Fig. 5 shows the reflection coefficient magnitude of a 40 ps-delay open-ended coplanar-waveguide line (not used for calibration), measured using LRM as well as LRRM. It can be seen that results are very similar. Therefore, the new LRRM theory and calibration algorithm are validated. The small differences observed are due to asymmetry in the match standards using LRM. Differences are more noticeable at high frequencies due to increasing asymmetry. Since LRRM requires only the measurement of a match in one of the two ports, significant accuracy improvements of LRRM calibration using the new algorithm, compared to LRM, are expected at higher frequencies (beyond $40 \mathrm{GHz}$ ).

\section{CONCLUSION}

In this paper a new theory of the LRRM calibration technique, that generalizes the three-standard self-calibration theory 
for VNA to four standards, has been presented. Using the criterion of equation-system condition-number, the optimum equation-system has been selected from experimental on-wafer data, to compute the coefficients of the eight-terms VNA error model.

A theoretical study on the reference impedance associated to an imperfect (actual) LRRM calibration has been developed, showing that the reference impedance is not defined whenever the line length is not zero. Therefore, a new method to compute the equivalent inductance $L_{\mathrm{e}}$ of the match standard model is proposed. Simulations show that the new method accurately computes the assumed $L_{\mathrm{e}}$, in contrast with a former method proposed in the literature.

Experimental on-wafer results demonstrate the feasibility of the new theoretical LRRM formulation. The measurement of a $40 \mathrm{ps}$ delay open-ended line shows the advantages of the LRRM algorithm versus LRM whenever asymmetries in the match occur, in particular at high frequencies.

\section{APPENDIX I}

Definition of Self-Calibration Measurement Matrices

Referring to Fig. 1, we have for the measured standard $i(i=$ $1,2,3,4,5)$

- In forward measurements:

$$
\left(\begin{array}{c}
b_{0} \\
a_{0}
\end{array}\right)=\frac{1}{e_{10} e_{32}}[\bar{X}] \cdot\left[T_{i}\right] \cdot[\bar{Y}] \cdot\left(\begin{array}{c}
a_{3} \\
b_{3}
\end{array}\right)
$$

- In reverse measurements:

$$
\left(\begin{array}{c}
b_{0}^{\prime} \\
a_{0}^{\prime}
\end{array}\right)=\frac{1}{e_{10} e_{32}}[\bar{X}] \cdot\left[T_{i}\right] \cdot[\bar{Y}] \cdot\left(\begin{array}{c}
a_{3}^{\prime} \\
b_{3}^{\prime}
\end{array}\right)
$$

where $[\bar{X}],[\bar{Y}]$ are defined in (1).

Combining (A1) and (A2), the measurement transmission matrix $\left[M_{i}\right]$ is defined [6]

$$
\left(\begin{array}{cc}
b_{0} & b_{0}^{\prime} \\
a_{0} & a_{0}^{\prime}
\end{array}\right) \cdot\left(\begin{array}{cc}
a_{3} & a_{3}^{\prime} \\
b_{3} & b_{3}^{\prime}
\end{array}\right)^{-1}=\frac{1}{e_{10} e_{32}}[\bar{X}] \cdot\left[T_{i}\right] \cdot[\bar{Y}]=\left[M_{i}\right]
$$

Developing (A3), the $S$-parameters corresponding to $\left[M_{i}\right]$, are expressed as

$$
\begin{aligned}
& S_{11}^{M_{i}}=\frac{\frac{b_{0}}{a_{0}}-\frac{a_{3} \cdot b_{0}^{\prime}}{a_{0} \cdot a_{3}^{\prime}}}{1-\frac{a_{0}^{\prime} \cdot a_{3}}{a_{3}^{\prime} \cdot a_{0}}} \quad S_{12}^{M_{i}}=\frac{\frac{b_{0}^{\prime}}{a_{3}^{\prime}}-\frac{a_{0}^{\prime} \cdot b_{0}}{a_{0} \cdot a_{3}^{\prime}}}{1-\frac{a_{0}^{\prime} \cdot a_{3}}{a_{3}^{\prime} \cdot a_{0}}} \\
& S_{21}^{M_{i}}=\frac{\frac{b_{3}}{a_{0}}-\frac{a_{3} \cdot b_{3}^{\prime}}{a_{0} \cdot a_{3}^{\prime}}}{1-\frac{a_{0}^{\prime} \cdot a_{3}}{a_{3}^{\prime} \cdot a_{0}}} \quad S_{22}^{M_{i}}=\frac{\frac{b_{3}^{\prime}}{a_{3}^{\prime}}-\frac{a_{0}^{\prime} \cdot b_{3}}{a_{0} \cdot a_{3}^{\prime}}}{1-\frac{a_{0}^{\prime} \cdot a_{3}}{a_{3}^{\prime} \cdot a_{0}}}
\end{aligned}
$$

with

$$
\left[M_{i}\right]=\frac{1}{S_{21}^{M_{i}}}\left(\begin{array}{cc}
S_{12}^{M_{i}} \cdot S_{21}^{M_{i}}-S_{11}^{M_{i}} \cdot S_{22}^{M_{i}} & S_{11}^{M_{i}} \\
-S_{22}^{M_{i}} & 1
\end{array}\right) .
$$

Normalized measurement matrices can also be defined from (A5) as $\left[\overline{M_{i}}\right]=\left[M_{i}\right] \cdot S_{21}^{M_{i}}$. In a similar way, normalized matrices of standards are defined as $\left[\overline{T_{i}}\right]=\left[T_{i}\right] \cdot S_{21}^{M_{i}}$. Using the $S$-parameter definition (A.4), the ANA internal switch is included in the measurement matrix and the eight-term error model holds for both directions. The number of required measurements to compute $\left[M_{i}\right]$ from (A4) is six, four standard ratios
$A, B, C, D$, and two additional measurements ( $E, F)$, defined as:

$$
\begin{array}{ll}
A=\frac{b_{0}}{a_{0}} \quad C=\frac{b_{0}^{\prime}}{a_{3}^{\prime}} \quad E=\frac{a_{3}}{a_{0}} \\
B=\frac{b_{3}}{a_{0}} \quad D=\frac{b_{3}^{\prime}}{a_{3}^{\prime}} \quad F=\frac{a_{0}^{\prime}}{a_{3}^{\prime}} .
\end{array}
$$

In case of dual one-port devices (reflect1, reflect2, matchreflect1, match-reflect2), $a_{o}^{\prime}=b_{0}^{\prime}=\mathrm{a}_{3}=b_{3}=0$, and (A4) reduces to

$$
\begin{aligned}
S_{11}^{M_{i}} & =\frac{b_{0}}{a_{0}} \quad S_{12}^{M_{i}}=0 \\
S_{21}^{M_{i}} & =0 \quad S_{22}^{M_{i}}=\frac{b_{3}^{\prime}}{a_{3}^{\prime}}
\end{aligned}
$$

and their normalized measurement matrices are $(i=2,3,4,5)$

$$
\left[\bar{M}_{i}\right]=\left(\begin{array}{cc}
-S_{11}^{M_{i}} \cdot S_{22}^{M_{i}} & S_{11}^{M_{i}} \\
-S_{22}^{M_{i}} & 1
\end{array}\right) .
$$

\section{APPENDIX II}

\section{EXPRESSION FOR THE COMPUTED REFLECTION COEFFICIENT OF REFLECT STANDARD}

In this appendix, the relationship between the computed reflection coefficient $\Gamma_{r 1}^{e}$ of standard reflect and its actual value $\Gamma_{r 1}$ is derived. The calibration algorithm assumes that standards match and line are perfectly matched, but they actually have a reflection coefficient different from zero $\left(\Gamma_{m} \neq 0\right.$ and $\left.\rho_{\mathrm{L}} \neq 0\right)$ referred to a given normalization impedance $Z_{o}$. Therefore, the actual line standard transmission matrix is

$$
\begin{aligned}
{\left[T_{1}\right] } & =\frac{1}{1-\left(\rho_{\mathrm{L}}\right)^{2}} \\
& \cdot\left(\begin{array}{cc}
e^{-\gamma \cdot l}-\left(\rho_{\mathrm{L}}\right)^{2} \cdot e^{\gamma \cdot l} & \rho_{\mathrm{L}} \cdot\left(e^{\gamma \cdot l}-e^{-\gamma \cdot l}\right) \\
-\rho_{\mathrm{L}} \cdot\left(e^{\gamma \cdot l}-e^{-\gamma \cdot l}\right) & e^{\gamma \cdot l}-\left(\rho_{\mathrm{L}}\right)^{2} \cdot e^{-\gamma \cdot l}
\end{array}\right) .
\end{aligned}
$$

Matrices $\left[\overline{T_{2}}\right]$ to $\left[\overline{T_{5}}\right][(18)-(21)]$ do not change. The normalized measurement matrices that VNA would measure can be computed using (15)

$$
\begin{aligned}
& {\left[\bar{M}_{21}\right]=[\bar{X}] \cdot\left[\bar{T}_{21}\right] \cdot[\bar{X}]^{-1}} \\
& {\left[\bar{M}_{31}\right]=[\bar{X}] \cdot\left[\bar{T}_{31}\right] \cdot[\bar{X}]^{-1}} \\
& {\left[\bar{M}_{51}\right]=[\bar{X}] \cdot\left[\bar{T}_{51}\right] \cdot[\bar{X}]^{-1}}
\end{aligned}
$$

where matrices $\left[\overline{T_{21}}\right],\left[\bar{T}_{31}\right],\left[\bar{T}_{51}\right]$ are computed using (17) $(i=$ $2,3,5)$ with $\left[T_{1}\right]$ given by (B.1). Since the line and the match standards are assumed perfectly matched, the unknown reflection coefficient of standard reflect $\Gamma_{r 1}^{e}$ is computed using (32) with $\Gamma_{M}=0$

$$
\Gamma_{r 1}^{e}= \pm e^{\gamma \cdot l} \cdot K_{\tilde{}}^{21} \cdot \sqrt{\frac{1-K_{31}^{21}}{K_{31}^{21} \cdot\left(2 \cdot K_{51}^{21}-\left(K_{51}^{21}\right)^{2}-1\right)}}
$$

where the definitions of $K_{31}^{21}, K_{51}^{21}$ are given in expressions (28) and (29). Substituting (28) and (29) into (B3), the following expression is obtained [see (B4) at the top of the following page]. 


$$
\Gamma_{r 1}^{e}= \pm \frac{\left(\Gamma_{m}-\Gamma_{r 1}\right) \cdot\left(\rho_{\mathrm{L}}^{2}-1\right)}{e^{2 \cdot \gamma \cdot l} \cdot\left(\rho_{\mathrm{L}}-\Gamma_{r 1}\right) \cdot\left(\rho_{\mathrm{L}}-\Gamma_{m}\right)+\left(\Gamma_{r 1} \cdot \rho_{\mathrm{L}}-1\right) \cdot\left(1-\Gamma_{m} \cdot \rho_{\mathrm{L}}\right)}
$$

\section{REFERENCES}

[1] R. A. Hackborn, "An automatic network analyzer system," Microwave J., pp. 45-52, May 1968.

[2] N. R. Franzen and R. A. Speciale, "A new procedure for system calibration and error removal in automated $S$-parameter measurements," in Proc. 5th Eur. Microwave Conf., Hamburg, Germany, Sept. 1-4, 1975, pp. 69-73.

[3] G. F. Engen and C. A. Hoer, "Thru-reflect-line: An improved technique for calibrating the dual six port automatic network analyzer," IEEE Trans. Microwave Theory Tech., vol. 27, no. 12, pp. 987-993, Dec. 1979.

[4] R. A. Soares, P. Gouzien, P. Legaud, and G. Follot, "A unified mathematical approach to two-port calibration techniques and some applications," IEEE Trans. Microwave Theory Tech., vol. 37, pp. 1669-1674, Nov. 1989.

[5] H.-J. Eul and B. Shieck, "Thru-match-reflect: One result of a rigorous theory for deembedding and network analyzer calibration," in 18th Eur. Microwave Conf., Stockholm, Sweden, 1988, pp. 909-914.

[6] - "A generalized theory and new calibration procedures for network analyzer self-calibration," IEEE Trans. Microwave Theory Tech., vol. 39, pp. 724-731, Apr. 1991.

[7] _ - "Reducing the number of calibration standards for network analyzer calibration," IEEE Trans. Instrum. Meas., vol. 40, pp. 732-735, Aug. 1991

[8] - "Robust algorithms for TXX VNA self-calibration procedures," IEEE Trans. Instrum. Meas., vol. 43, pp. 18-23, Feb. 1994.

[9] L. Pradell, F. Purroy, and M. Cáceres, "Development of self-calibration techniques for on- wafer and fixtured measurements: a novel approach," in 22nd Eur. Microwave Conf. Proc., Sept. 1992, pp. 919-924.

[10] A. Davidson, K. Jones, and E. Strid, "LRM and LRRM calibrations with automatic determination of load inductance," in 36th ARTFG Conf. Dig., Nov. 1990, pp. 57-63.

[11] T. T. Ha, Solid-state Microwave Amplifier Design, New York: Wiley, 1981, ch. 2, pp. 33-34.
[12] D. F. Williams and R. B. Marks, "Comparison of on-wafer calibrations," in 38th ARTFG Conf. Dig., Dec. 1991, pp. 68-81.

[13] L. Pradell and F. Purroy, "Comparison of on-wafer calibrations using the concept of reference impedance," in 23rd Eur. Microwave Conf. Proc., Sept. 1993, pp. 857-859.

[14] F. Purroy, "Tècniques de caracterització de transistors de microones amb aplicació al seu modelatge lineal," Ph.D. (in Catalan and in Spanish), Univ. Politècnica de Catalunya, Barcelona, Spain, June 1996.

Francesc Purroy was born in Barcelona, Catalunya, Spain, in 1965. He received the telecommunication engineer degree in 1989 and the doctor degree in 1996, both from the Universitat Politècnica de Catalunya (UPC), Barcelona, Spain.

His main areas of research have been on-wafer MESFET transistors characterization and network analyzer calibration techniques. His research interest includes transistor modeling and Power Amplifier linearization. In 1997, he joined Ericsson Radio Access AB in Kista, Stockholm, Sweden. He is now working there as Senior Specialist in MCPA (Multi Carrier Power Amplifier) design.

Lluís Pradell was born in Barcelona, Catalunya, Spain, in 1956. He received the telecommunication engineering degree from Universitat Politècnica de Catalunya (UPC), Barcelona, in 1981. In 1985, he joined the faculty at UPC, where he received the doctorate degree in 1989.

From 1981 to 1985, he worked at Mier Allende, S.A., Barcelona, as an RF \& Microwave System Design Engineer. Since 1985, he has been teaching courses on microwave circuits and antennas, and performing research on microwave and millimeter-wave devices and systems (such as $40 \mathrm{GHz}$ MVDS transceivers), on-wafer network analyzer calibration techniques, noise parameter determination, and modeling of MESFETs and HEMTs. 